\title{
On free discrete subgroups of $\operatorname{Diff}(I)$
}

\author{
AZER AKHMEDOV
}

We prove that the free group $\mathbb{F}_{2}$ admits a faithful discrete representation into Diff $_{+}^{1}[0,1]$. We also prove that $\mathbb{F}_{2}$ admits a faithful discrete representation of biLipschitz class into $\mathrm{Homeo}_{+}[0,1]$. Some properties of these representations are studied.

37E05; 20F65

\section{Introduction}

In recent decades and especially in recent years, some remarkable papers devoted to the study of finitely generated subgroups of Diff ${ }_{+}^{1}[0,1]$ have appeared (see Bergman [1], Calegari [2], Farb and Franks [3; 4], Farb and Shalen [5], Ghys [6], Navas [7; 8; 9], Tsuboi [12] and Yoccoz [13] for some of the most current developments). In contrast, discrete subgroups of Diff ${ }_{+}^{1}[0,1]$ are much less studied. Very little is known in this area especially in comparison with the very rich theory of discrete subgroups of Lie groups started in the works of F Klein and H Poincare in the 19th century, and expanded enormously in the works of A Selberg, A Borel, G Mostow, G Margulis and many others in the 20 th century. Many questions which are either very easy or were studied a long time ago for (discrete) subgroups of Lie groups remain open in the context of the infinite-dimensional group $\operatorname{Diff}_{+}^{1}[0,1]$ and its relatives. In this paper, we address a question about the existence of discrete faithful representations of nonabelian free groups into the group Diff ${ }_{+}^{1}[0,1]$.

We assume the usual topology on the group $\operatorname{Diff}_{+}^{1}[0,1]$ given by the standard metric of $C^{1}[0,1]$. We will denote this metric by $d_{1}$.

Theorem 1 A free group $\mathbb{F}_{2}$ admits a faithful discrete representation into Diff ${ }_{+}^{1}[0,1]$.

We will also be interested in discrete subgroups of Homeo+ $[0,1]-$ the group of orientation preserving homeomorphisms of the closed interval. Here, the metric comes from the sup norm of the Banach space $C[0,1]$. For $f \in C[0,1]$ we will denote $\|f\|_{0}=\sup _{x \in[0,1]}|f(x)|$. 
Theorem 2 A free group $\mathbb{F}_{2}$ admits a faithful discrete representation into $\mathrm{Homeo}_{+}[0,1]$. Moreover,

(a) the representation can be chosen from the class $C^{1}(0,1) \cap \operatorname{BiLip}[0,1]$.

(b) for any nonempty open neighborhood $\Omega$ of the identity in Homeo+ $[0,1]$, the generators of the faithful discrete representation of $\mathbb{F}_{2}$ can be chosen from $\Omega$.

Here, BiLip $[0,1]$ denotes the set of all bi-Lipschitz functions from the closed interval $[0,1]$ into itself.

\section{Proofs of main theorems}

In this section we will prove Theorems 1 and 2.

In the free group $\mathbb{F}_{2}$ we will fix the left-invariant Cayley metric with respect to standard generating set, and denote it by $|\cdot|$. The following notions will be useful.

Definition 1 Let $W$ be a reduced word in the alphabet of the standard generating set of the free group $\mathbb{F}_{2}$. We say that a reduced word $U$ is a suffix of $W$, if $W=U_{1} U$ where $U_{1}$ is a reduced word, and $|W|=\left|U_{1}\right|+|U|$. We also say that a reduced word $V$ is a prefix of $W$, if $W=V V_{1}$ where $V_{1}$ is a reduced word, and $|W|=|V|+\left|V_{1}\right|$.

Proof of Theorem 1 Let $I_{n}=(1 /(2 n+1), 1 /(2 n))$ for any $n \in \mathbb{N}$ and let $C>0$.

We will build two maps $f, g \in \operatorname{Diff}_{+}^{1}[0,1]$ such that the group $\Gamma_{f, g}$ generated by them is isomorphic to $\mathbb{F}_{2}$ and satisfies the following condition:

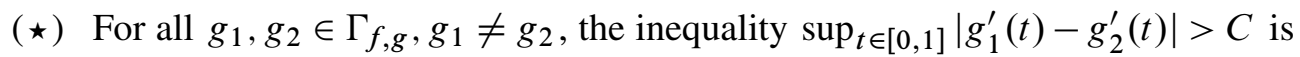
satisfied.

Let $\pi_{n}=\left(U_{n}, V_{n}\right), n \geq 1$ be a sequence of pairs of words (elements) in $\mathbb{F}_{2}$ satisfying the following conditions:

(a1) $U_{n} \neq V_{n}$ for all $n \geq 1$.

(a2) $\left|U_{n}\right| \geq\left|V_{n}\right|$ for all $n \geq 1$.

(a3) If $m>n$ then $\left|U_{m}\right| \geq\left|U_{n}\right|$.

(a4) If $m>n,\left|U_{m}\right|=\left|U_{n}\right|$ then $\left|V_{m}\right| \geq\left|V_{n}\right|$.

(a5) $U_{n} \neq 1$ for all $n \geq 1$.

(a6) If $U, V \in \mathbb{F}_{2}, U \neq 1,|U| \geq|V|$ then there exists $n \in \mathbb{N}$ such that $U=U_{n}, V=V_{n}$.

(a7) If $m \neq n$ then $\pi_{m} \neq \pi_{n}$. 
For every $n \in \mathbb{N}$, the longest common suffix of $U_{n}$ and $V_{n}$ will be denoted by $W_{n}$ and we let $s_{n}=\left|W_{n}\right|$.

Let also $m_{n}=\operatorname{Card}\left\{k\left|\pi_{k}=\left(U_{k}, V_{k}\right),\right| U_{k} \mid=n\right\}$ for all $n \geq 1, m_{0}=0$. Notice that $m_{n}$ grows exponentially as $n \rightarrow \infty$.

Let $\alpha=\left(\alpha_{1}, \alpha_{2}, \ldots\right)$ be a sequence of positive real numbers such that

(b1) $\lim _{r \rightarrow \infty} \alpha_{r}=0$.

(b2) for every $r \in \mathbb{N}, s \in\{0,1, \ldots, r-1\}$, the inequality

$$
\left(1+\alpha_{r}\right)^{s}\left(\left(1+\alpha_{r}\right)^{r-s}-1\right)>C
$$

is satisfied.

(Notice that such a sequence $\alpha$ exists, eg $\alpha_{1}=C+1, \alpha_{r}=\sqrt{(C+1) /(r-1)}, r \geq 2$.) Let also $\beta=\left(\beta_{1}, \beta_{2}, \ldots\right)$ be a sequence such that $\beta_{i}=\alpha_{j}$ for all $m_{1}+\cdots+m_{j-1}<$ $i \leq m_{1}+\cdots+m_{j-1}+m_{j}$. We notice that $\lim _{n \rightarrow \infty} \beta_{n}=0$; moreover, for every $n \in \mathbb{N}$, we have $\beta_{n}=\alpha_{i(n)}$ where $i(n) \rightarrow \infty$ as $n \rightarrow \infty$.

Now, for any natural $n$, let $x_{0}^{n}$ be the midpoint of the interval $I_{n}, s=s_{n}$, and let $f, g$ be defined in the interval $I_{n}$ such that

(c1) $f(x)=g(x)=x$ for all $x \in\{1 /(2 n+1), 1 /(2 n)\}$.

(c2) $f^{\prime}(x) \in\left[1 /\left(1+\beta_{n}+1 / n\right), 1+\beta_{n}+1 / n\right]$, for all $x \in I_{n}$.

(c3) $f^{\prime}(x)=g^{\prime}(x)=1$ for all $x \in\{1 /(2 n+1), 1 /(2 n)\}$.

(c4) if $\left|U_{n}\right|=r$, where $U_{n}=a_{r} a_{r-1} \cdots a_{s} \cdots a_{1}$ for $a_{i} \in\left\{f, g, f^{-1}, g^{-1}\right\}, 1 \leq i \leq r$, and if $U_{n}(k)=a_{k} \cdots a_{1}, 0 \leq k \leq r-1$, then $a_{k+1}^{\prime}\left(U_{n}(k)\left(x_{0}^{n}\right)\right)=1+\beta_{n}$.

(c5) if $\left|V_{n}\right|=m$, where $V_{n}=b_{m} b_{m-1} \cdots b_{1}$, for $b_{i} \in\left\{f, g, f^{-1}, g^{-1}\right\}, 1 \leq i \leq m$, and if $V_{n}(k)=b_{k} \cdots b_{1}, m-1 \geq k \geq s$ then $b_{k+1}^{\prime}\left(V_{n}(k)\left(x_{0}^{n}\right)\right)=1$.

Now, if $x \in[0,1] \backslash\left(\bigsqcup_{n \in \mathbb{N}} I_{n}\right)$, we set $f(x)=g(x)=x$ (hence $\left.f^{\prime}(x)=g^{\prime}(x)=1\right)$.

Then the functions $f, g$ will belong to $\operatorname{Diff}_{+}^{1}[0,1]$. Moreover, for any $n \geq 1$, by the Chain Rule, we have

$$
U_{n}^{\prime}\left(x_{0}^{n}\right)=\left(1+\beta_{n}\right)^{r}, \quad V_{n}^{\prime}\left(x_{0}^{n}\right)=\left(1+\beta_{n}\right)^{s} 1^{m-s}=\left(1+\beta_{n}\right)^{s} .
$$

Since $\beta_{n}=\alpha_{i(n)}$ and $i(n)=r$, the inequality $\left|\left(U_{n}(f, g)\right)^{\prime}\left(x_{0}^{n}\right)-\left(V_{n}(f, g)\right)^{\prime}\left(x_{0}^{n}\right)\right|>C$ follows from condition (b2). 
Remark 1 We indeed prove more than discreteness; the inequality

$$
\sup _{\substack{t \in[0,1], g \in \mathbb{F}_{2} \backslash\{1\}}}\left|g^{\prime}(t)-1\right| \geq C>0
$$

would suffice for discreteness. By proving more general inequality

$$
\sup _{\substack{t \in[0,1], g_{1}, g_{2} \in \mathbb{F}_{2}, g_{1} \neq g_{2}}}\left|g_{1}^{\prime}(t)-g_{2}^{\prime}(t)\right| \geq C>0,
$$

we show that the representation is uniformly discrete. Since the metric in Diff $\operatorname{Di}_{+}^{1}[0,1]$ is not left-invariant, discreteness does not necessarily imply uniform discreteness.

Remark 2 It is clear from the proof that the functions $f(t)$ and $g(t)$ can be chosen from an arbitrary nonempty open neighborhood of the identity. This is contrary to the case of connected Lie groups: the Margulis Lemma states that any connected Lie group $G$ possesses a nonempty open neighborhood $U$ of the identity such that any discrete subgroup of $G$ generated by elements from $U$ is nilpotent (see Raghunathan [10]). Thus we have shown that the Margulis Lemma does not hold for the group Diff ${ }_{+}^{1}[0,1]$.

It is easy to put the main idea of the proof of Theorem 1 in words: we take all pairs $\left(U_{n}, V_{n}\right)$ in the free group $\mathbb{F}_{2}$ that are interesting to us and enumerate them with some care (conditions (a1)-(a7)). For simplicity, let us also assume that $V_{n}=1, n \geq 1$. Then we choose countable pairwise disjoint open subintervals $I_{1}, I_{2}, \ldots, I_{n}, \ldots$ of $[0,1]$ which are accumulating to the left endpoint of [0,1], ( $I_{i}$ is on the left side of $I_{j}$ for all $i>j$ ). Then, on each of the subintervals we arrange the maps $f, g$ such that $\sup _{x \in I_{n}}\left|f^{\prime}(x)-1\right|$ and $\sup _{x \in I_{n}}\left|g^{\prime}(x)-1\right|$ converge to zero as $n \rightarrow \infty$ while for each midpoint $x_{n} \in I_{n}$ we have $U_{n}^{\prime}\left(x_{n}\right)>C$.

To satisfy this condition, one notices that the word $U_{n}$ has length at least $\log (n)$ which goes to infinity as $n$ grows. Then, since $U_{n}^{\prime}\left(x_{n}\right)$ is the product of $\log (n)$ derivatives we can have this product to be bigger than $C$ yet each of the factor stay close to 1 . (and converge to 1 as $n$ goes to infinity). For fixed $n$, each of these conditions imposes only finitely many conditions on $f$ and $g$ in $I_{n}$, and for the next pair we go to a different interval $I_{n+1}$, hence we have no obstruction left to the existence of discrete $\mathbb{F}_{2}$ of $C^{1}$ class.

However, because of the slow growth of $\log (n)$, and because the lengths of intervals of $I_{n}$ converge to zero faster than $1 / n$, it is easy to see that this construction will not work in $C^{2}$ class. In fact, as Danny Calegari pointed out, it will not work in any $C^{1+\epsilon}$ class for any $\epsilon>0$; imposing the same condition will blow-up the Holder norm. So one cannot achieve higher regularity of representations by taking care of different 
pairs in disjoint areas of the closed interval $[0,1]$. If we want to mix fields of actions for different pairs, we need to take some cautions.

Now we will prove Theorem 2. We need the following definitions.

Definition 2 For open subintervals $I, J \subset(0,1)$ we say $I<J$ if any element $I$ is less than any element of $J$.

Definition 3 A two-sided sequence $\left\{I_{n}\right\}_{n \in \mathbb{Z}}$ of open subintervals of $(0,1)$ is called a chain if $I_{n}<I_{n+1}$ for all $n \in \mathbb{Z}$.

Proof of Theorem 2 Let $\epsilon>0$, and let $A_{n}, B_{n}, n \in \mathbb{Z}$ be open subintervals of $(0,1)$ such that

(i) the two sided sequence $\left\{A_{n}, B_{n}\right\}_{n \in \mathbb{Z}}$ is a chain of subintervals (that is, we have $\left.\cdots<A_{-1}<B_{-1}<A_{0}<B_{0}<A_{1}<B_{1}<A_{2}<\cdots\right)$.

(ii) for all $n \in \mathbb{Z}$ and all $i \in\{1,2,3,4\}$ we have $f^{i}\left(A_{n}\right) \subseteq B_{n}, f^{-i}\left(A_{n}\right) \subseteq B_{n-1}$.

(iii) for all $n \in \mathbb{Z}$, we have $g\left(B_{n}\right) \subseteq A_{n+1}, g^{-1}\left(B_{n}\right) \subseteq A_{n}$.

(iv) for all $n \in \mathbb{Z}$, the inequality $\sup _{x \in A_{n}, y \in A_{n+2}}|x-y|<\epsilon$ holds.

It is straightforward to choose $f, g \in$ Homeo $_{+}[0,1]$ satisfying conditions (i)-(iv).

Now, let $A=\bigcup_{n \in Z} A_{n}, B=\bigcup_{n \in Z} B_{n}$. Notice that by conditions (i)-(ii),

$f^{i}(A) \subseteq B$ for all $i \in\{-4,-3,-2,-1,1,2,3,4\}$ and $g^{i}(B) \subseteq A$ for all $i \in\{-1,1\}$.

This allows us to use a ping-pong argument.

The ping-pong argument is usually used to guarantee existence of free subgroups, here we will be using it also to satisfy discreteness (which is natural). Using the pingpong lemma, we will show the following: Assume conditions (i)-(iv), and suppose $U(f, g), V(f, g)$ are reduced words satisfying two conditions:

(1) $U(f, g)=f^{2} U_{0}(f, g) f^{2}, V(f, g)=f V_{0}(f, g) f$ where $U_{0}(f, g), V_{0}(f, g)$ are both nonempty reduced words starting and ending in letter $g$.

(2) None of the letters $\{f, g\}$ occur with exponent other than $\{-1,1\}$ in $U_{0}(f, g)$ and in $V_{0}(f, g)$.

Then $U(f, g)$ and $V(f, g)$ actually generate a free subgroup isomorphic to $\mathbb{F}_{2}$ in Homeo $_{+}[0,1]$. We will have that this subgroup (which we will denote by $\Gamma$ ) is discrete. 
Let $W(U, V)$ be any reduced nontrivial word in the alphabet $\{U=U(f, g), V=$ $\left.V(f, g), U^{-1}=U(f, g)^{-1}, V^{-1}=V(f, g)^{-1}\right\}$. Then in the alphabet $\left\{f, g, f^{-1}, g^{-1}\right\}$ the word $W$ ends with either $f$ or $f^{-1}$.

Let $x_{0}$ be the midpoint of $A_{0}$.

We notice that $f^{i}(A) \subseteq B$ for all $i \in\{-4,-3,-2,-1,1,2,3,4\}$. Furthermore, $g^{ \pm 1}(B) \subseteq A$. Then by a standard ping-pong argument, we have that

$$
W\left(x_{0}\right)=W(U(f, g), V(f, g))\left(x_{0}\right) \notin A_{0},
$$

hence $W \neq 1$ in $\Gamma$, and $\|W\|_{0} \geq\left|A_{0}\right| / 2$.

We now consider the general case of arbitrary distinct $h_{1}, h_{2} \in \Gamma$. Let $h_{1}=W_{1}(U, V)$, $h_{2}=W_{2}(U, V)$ be two distinct reduced words in the alphabet $\left\{U, V, U^{-1}, V^{-1}\right\}$. Then we can write $W_{2}=W W_{1}$ where $W=W(U, V)=W(U(f, g), V(f, g))$.

Since $W_{1}=W_{1}(U(f, g), V(f, g))$ is a bijective map from [0,1] onto [0,1], there exists $z \in[0,1]$ such that $W_{1}(z)=x_{0}$. Then $W_{2}(z)=W\left(W_{1}(z)\right)=W\left(x_{0}\right) \notin A_{0}$.

Then we have $\left|W_{1}(z)-W_{2}(z)\right|=\left|x_{0}-W\left(x_{0}\right)\right|>\left|A_{0}\right| / 2$. Thus we established that the nonabelian free subgroup generated by $U$ and $V$ is discrete.

For claim (b), suppose $\Omega$ contains a ball of radius $r$, and $M=\max \{|U|,|V|\}$. Then by condition (iv), $\max \left\{\|U\|_{0},\|V\|_{0}\right\}<\epsilon M$. Since $\epsilon$ is arbitrary we can choose it to be such that $M \epsilon<r$, and hence we obtain claim (b).

For claim (a), we may choose a sufficiently large natural number $N$, and further assume that

$$
\begin{array}{ll}
A_{n}=\left(\frac{1}{5(|n|+1)}, \frac{1}{5|n|+4}\right), B_{n}=\left(\frac{1}{5|n|+4}, \frac{1}{5|n|}\right) & \text { for all } n \leq-N, \\
A_{n}=\left(1-\frac{1}{5 n}, 1-\frac{1}{5 n+1}\right), \quad B_{n}=\left(1-\frac{1}{5 n+1}, 1-\frac{1}{5(n+1)}\right) & \text { for all } n \geq N
\end{array}
$$

(and we choose $A_{-N+1}, B_{-N+1}, \ldots, A_{N-1}, B_{N-1}$ to be arbitrary open nonempty intervals such that conditions (i) and (iv) hold). Then it is straightforward to choose $f, g \in$ Homeo $_{+}[0,1]$ such that $f \in C^{1}[0,1], g \in C^{1}(0,1)$, and $g$ is a bi-Lipschitz function with Lipschitz constant at most 5 in $[0,1 /(5 N)]$ and in $[1-1 /(5 N), 1]$. Then for any word $W$ in the free group $\mathbb{F}_{2}$, the function $W(U(f, g), V(f, g))$ will be a bi-Lipschitz function of class $C^{1}(0,1)$.

Remark 3 We would like to point out what goes wrong if one applies the idea of the proof to Theorem 2 directly to obtain a faithful discrete representation of $\mathbb{F}_{2}$ in $\operatorname{Diff}_{+}^{1}[0,1]$ : 
Let $A_{n}, B_{n}, n \in \mathbb{Z}$ be mutually disjoint open subintervals in $(0,1)$ satisfying conditions (i), (ii) and (iii).

We will show that it is impossible to have the maps differentiable ( $C^{1}$ class) under these conditions (i)-(iii); there are obstructions easily obtained from the Mean Value Theorem.

Without loss of generality we may assume that $A_{n}, B_{n}$ converge to 1 as $n \rightarrow \infty$. Let $\lim _{x \rightarrow 1^{-}} f^{\prime}(x)=p$. (Then $p>0$.)

Let $p_{1}, p_{2}$ be positive real numbers such that

$$
p_{1}<p<p_{2}, \quad p_{1}>\frac{99}{100} p, \quad p_{2}<\frac{101}{100} p .
$$

So by the Mean Value Theorem, from condition (ii) we obtain that

$$
\left|B_{n}\right|>\left(p_{1}+p_{1}^{2}+p_{1}^{3}\right)\left|A_{n}\right| \text { and }\left|B_{n}\right|>\left(\frac{1}{p_{2}}+\frac{1}{p_{2}^{2}}+\frac{1}{p_{2}^{3}}\right)\left|A_{n+1}\right|
$$

for sufficiently big positive $n$. Then

$$
\begin{aligned}
& \frac{\left|g\left(B_{n}\right)\right|}{\left|B_{n}\right|} \leq \frac{\left|A_{n+1}\right|}{\left|B_{n}\right|}<\frac{1}{1 / p_{2}+1 / p_{2}^{2}+1 / p_{2}^{3}} \\
& \frac{\left|g^{-1}\left(B_{n}\right)\right|}{\left|B_{n}\right|} \leq \frac{\left|A_{n}\right|}{\left|B_{n}\right|}<\frac{1}{p_{1}+p_{1}^{2}+p_{1}^{3}} .
\end{aligned}
$$

Then, by the Mean Value Theorem, we obtain that for sufficiently big positive $n$, there exists $u_{n}, v_{n} \in B_{n}$ such that

$$
g^{\prime}\left(u_{n}\right)<\frac{1}{1 / p_{2}+1 / p_{2}^{2}+1 / p_{2}^{3}} \quad \text { and } \quad\left(g^{-1}\right)^{\prime}\left(v_{n}\right)<\frac{1}{p_{1}+p_{1}^{2}+p_{1}^{3}} .
$$

However, since $\lim _{x \rightarrow 1^{-}} g^{\prime}(x)=1 / \lim _{x \rightarrow 1^{-}}\left(g^{-1}\right)^{\prime}(x)$, we obtain a contradiction because

$$
\frac{1}{1 / p_{2}+1 / p_{2}^{2}+1 / p_{2}^{3}} \frac{1}{p_{1}+p_{1}^{2}+p_{1}^{3}}<\frac{1}{p_{1} / p_{2}+p_{1}^{2} / p_{2}^{2}+p_{1}^{3} / p_{2}^{3}}<\frac{1}{2}<1 .
$$

Remark 4 In the proof of Theorem 2, by slightly changing conditions (1)-(2), it is possible to replace condition (ii) by the following weaker version:

(ii) $^{\prime}$ for all $i \in\{1,2\}, n \in \mathbb{Z}$, we have $f^{i}\left(A_{n}\right) \subseteq B_{n}, f^{-i}\left(A_{n}\right) \subseteq B_{n-1}$.

However, a similar argument shows that there are no $f, g \in \operatorname{Diff}_{+}^{1}[0,1]$ satisfying conditions (i), (ii)' and (iii). It also follows from the criterion of Calegari [2] that no $C^{1}$-class diffeomorphisms exist which satisfy conditions (i), (ii) ${ }^{\prime}$ and (iii). 
Remark 5 The metric in $C^{1}[0,1]$ is given by the norm $\|f\|=\|f\|_{0}+\|f\|_{1}$ where $\|f\|_{0}=\sup _{x \in[0,1]}|f(x)|,\left|\|f\|_{1}=\sup _{x \in[0,1]}\right| f^{\prime}(x) \mid$. If $\mid\|f\|_{1}$ is small and $f(0)=0$, then by Mean Value Theorem $\mid\|f\|_{0}$ cannot be big. However, $\mid\|f\|_{1}$ can be big even if $\mid\|f\|_{0}$ is small. In the proof of Theorem 1, taking $f(x)=W(x)-x$, we actually show that $\mid\|f\|_{1}$ stays big for all $W \neq 1$; we do not show that $\mid\|f\|_{0}$ is big. However, in the proof of Theorem 2, we indeed show a stronger fact that $\mid\|f\|_{0}$ remains big.

\section{Questions}

In this section, we raise several questions. We will address these questions in our next article.

The regularity of the representation is a very interesting question; if a finitely generated group $\Gamma$ admits a faithful discrete representation in $\operatorname{Diff}_{+}^{1}[0,1]$ or in Homeo $+[0,1]$, it is interesting to know if one can achieve faithful discrete representations of higher $\left(C^{k}, k>1, C^{\infty}\right.$, analytic, etc) regularity.

Question 1 Does a free group $\mathbb{F}_{2}$ admit a faithful discrete representation into $\operatorname{Diff}_{+}^{1}[0,1]$

(a) of $C^{k}$ regularity for some $k>1$ ?

(b) of $C^{k}$ regularity for any $k \geq 1$ ?

(c) of $C^{\infty}$ regularity?

(d) of analytic regularity?

Let $\Gamma$ be a finitely generated group, and $\pi: \Gamma \rightarrow \operatorname{Diff}_{+}^{1}[0,1]$ be a faithful discrete representation of it.

Definition 4 The representation $\pi$ is called $\|\cdot\|_{0}$-discrete if there exists $C>0$ such that $\|\pi(g)\|_{0}>C$ for all $g \in \Gamma \backslash\{1\}$.

By Remark 5, $\|\cdot\|_{0}$-discreteness of the representation implies its discreteness in $\operatorname{Diff}_{+}^{1}[0,1]$. Also, a $\|\cdot\|_{0}$-discrete representation of a group into $\operatorname{Diff}_{+}^{1}[0,1]$ is just a discrete representation into Homeo+ $[0,1]$ of $C^{1}$-regularity.

Question 2 Does $\mathbb{F}_{2}$ admit a faithful $\|\cdot\|_{0}$-discrete representation into Diff ${ }_{+}^{1}[0,1]$ ?

Definition 5 The representation $\pi$ is called strongly discrete if there exists $C>0$ and $x_{0} \in(0,1)$ such that $\left\|\pi(g)\left(x_{0}\right)\right\|_{1}>C$ for all $g \in \Gamma \backslash\{1\}$. 
Question 3 Does $\mathbb{F}_{2}$ admit a faithful strongly discrete representation into Diff ${ }_{+}^{1}[0,1]$ ?

Similarly, we say that a faithful representation $\pi: \Gamma \rightarrow$ Homeo $_{+}[0,1]$ is strongly discrete (in Homeo+ $[0,1]$ ) if there exists $C>0$ and $x_{0} \in(0,1)$ such that $\left\|\pi(g)\left(x_{0}\right)\right\|_{0}>C$ for all $g \in \Gamma \backslash\{1\}$. Notice that in the proof of Theorem 2, the representation of $\mathbb{F}_{2}$ into $\mathrm{Homeo}_{+}[0,1]$ is indeed strongly discrete.

Definition 6 Let $G$ be a topological group or a group with a metric. We say that the Weak Margulis Lemma holds for $G$, if there exists an open nonempty neighborhood $U$ of identity such that any discrete subgroup of $G$ generated by elements from $U$ does not contain a nonabelian free subgroup.

We will be interested in the group $\operatorname{Diff}_{+}^{1+\epsilon}[0,1]$ where $\epsilon$ is a fixed positive real number. On this group, we are considering the metric $d_{1}$, ie the metric which comes from the Banach norm of $C^{1}[0,1]$.

Question 4 Does the Weak Margulis Lemma hold for the group Diff ${ }_{+}^{1+\epsilon}[0,1]$ for some $\epsilon>0$ ?

Remark 6 It follows from the proof of Theorem 1 and from Theorem 2 that the Weak Margulis Lemma does not hold neither for Diff ${ }_{+}^{1}[0,1]$ nor for Homeo+ $[0,1]$, in respective metrics.

The study of discrete subgroups of $\operatorname{Diff}_{+}^{1}[0,1]$ is interesting beyond the existence question of discrete faithful representations of free groups or even of the groups which contain nonabelian free subgroups. The existence of a faithful representation into $\operatorname{Diff}_{+}^{1}[0,1]$ imposes some algebraic properties onto the group; for example, it is wellknown that any subgroup of Homeo+ $[0,1]$ is left-orderable (see Ghys [6]). Furthermore, if a group is isomorphic to a subgroup of Diff ${ }_{+}^{1}[0,1]$ then it is locally indicable, as proven by Thurston [11]. It is interesting to consider if discreteness implies further algebraic restrictions on the group. We would like to ask the following:

Question 5 Is there a finitely generated group which admits a faithful representation into Diff $+[0,1]$ but does not admit a faithful discrete representation?

Acknowledgments I am thankful to Matthew G Brin and Danny Calegari for useful discussions related to the content of this paper. I also would like to thank to two anonymous referees for valuable comments. 


\section{References}

[1] G M Bergman, Right orderable groups that are not locally indicable, Pacific J. Math. 147 (1991) 243-248 MR1084707

[2] D Calegari, Nonsmoothable, locally indicable group actions on the interval, Algebr. Geom. Topol. 8 (2008) 609-613 MR2443241

[3] B Farb, J Franks, Group actions on one-manifolds. II. Extensions of Hölder's theorem, Trans. Amer. Math. Soc. 355 (2003) 4385-4396 MR1986507

[4] B Farb, J Franks, Groups of homeomorphisms of one-manifolds. III. Nilpotent subgroups, Ergodic Theory Dynam. Systems 23 (2003) 1467-1484 MR2018608

[5] B Farb, P Shalen, Groups of real-analytic diffeomorphisms of the circle, Ergodic Theory Dynam. Systems 22 (2002) 835-844 MR1908556

[6] É Ghys, Groups acting on the circle, Enseign. Math. (2) 47 (2001) 329-407 MR1876932

[7] A Navas, Sur les groupes de difféomorphismes du cercle engendrés par des éléments proches des rotations, Enseign. Math. (2) 50 (2004) 29-68 MR2084334

[8] A Navas, Growth of groups and diffeomorphisms of the interval, Geom. Funct. Anal. 18 (2008) 988-1028 MR2439001

[9] A Navas, A finitely generated, locally indicable group with no faithful action by $C^{1}$ diffeomorphisms of the interval, Geom. Topol. 14 (2010) 573-584 MR2602845

[10] MS Raghunathan, Discrete subgroups of Lie groups, Ergebnisse der Math. und ihrer Grenzgebiete 68, Springer, New York (1972) MR0507234

[11] W P Thurston, A generalization of the Reeb stability theorem, Topology 13 (1974) 347-352 MR0356087

[12] T Tsuboi, Homological and dynamical study on certain groups of Lipschitz homeomorphisms of the circle, J. Math. Soc. Japan 47 (1995) 1-30 MR1304186

[13] J-C Yoccoz, Centralisateurs et conjugaison différentiable des difféomorphismes du cercle. Petits diviseurs en dimension 1, Astérisque 231, Soc. Math. France (1995) MR1367354

Department of Mathematics, North Dakota State University Fargo ND 58102, USA

azer.akhmedov@ndsu.edu

Received: 26 April 2010 Revised: 14 September 2010 